\title{
A GAS SCINTILLATION PROPORTIONAL DETECTOR TO SEARCH FOR 17 KEV NEUTRINOS
}

\author{
W.J.C. Okx; V.R. Bom, C.W.E. van Eijk, R.W. Hollander. \\ Faculty of Applied Physics, Radiation Technology Group, \\ Delft University of Technology, Delft, \\ The Netherlands.
}

\begin{abstract}
Evidence for the existence of a $17 \mathrm{keV}$ neutrino was first reported in 1985 . Since then many experiments have been performed with contradicting results.

In this paper we describe an experiment with a new approach to the problem by the introduction of a Gas Scintillation Proportional Detector.
\end{abstract}

\section{Introduction}

In 1985 John Simpson at the University of Guelph in Ontario reported a distortion in the beta decay spectrum of tritium $17 \mathrm{keV}$ below the endpoint energy. This was considered evidence for the existence of a neutrino weighing $17 \mathrm{keV}$ [1].

The "standard model" of the fundamental interactions of elementary particles presumes that the masses of all the neutrino species are sero. One can easily get non-vanishing neutrino masses from modest and plausible extensions of the model. However, the existence of a $17 \mathrm{keV}$ neutrino would require a serious revision of the theory of interactions of elementary particles.

Many experiments have been performed to check Simpson's result. Some confirm the reported distortion, oth- ers exclude it to a high degree of accuracy [2], [3]. The general tendency of beta decay measurements is that semi-conductor detector experiments confirm the $17 \mathrm{keV}$ neutrino observation, apart from some incidental cases, whereas magnetic spectrometer results are negative. Obviously systematic errors play an important role. This is also the case for the IBEC data (positive and negative results) and a proportional counter experiment on tritium, with a negative result [4].

With a gas scintillation proportional detector (GSPD) it is possible to check the original experiment with an energy resolution of the same magnitude and with the benefits of its calorimetric approach. In this detector however, possible disturbing solid state phenomena from tritium in the semiconductor lattice are excluded.

\section{The detector}

A schematic drawing of the detector is shown in figure 1 . The aluminium house of the detector consists of three parts. The upper part contains the gas scintillator and a high voltage (HV) bleeder to define a smoothly increasing potential within the scintillator. This part is flushed with $\mathrm{CO}_{2}$ to prevent electrical breakdown.

*Fellow of the Royal Netherlands Academy of Arts and Sciences

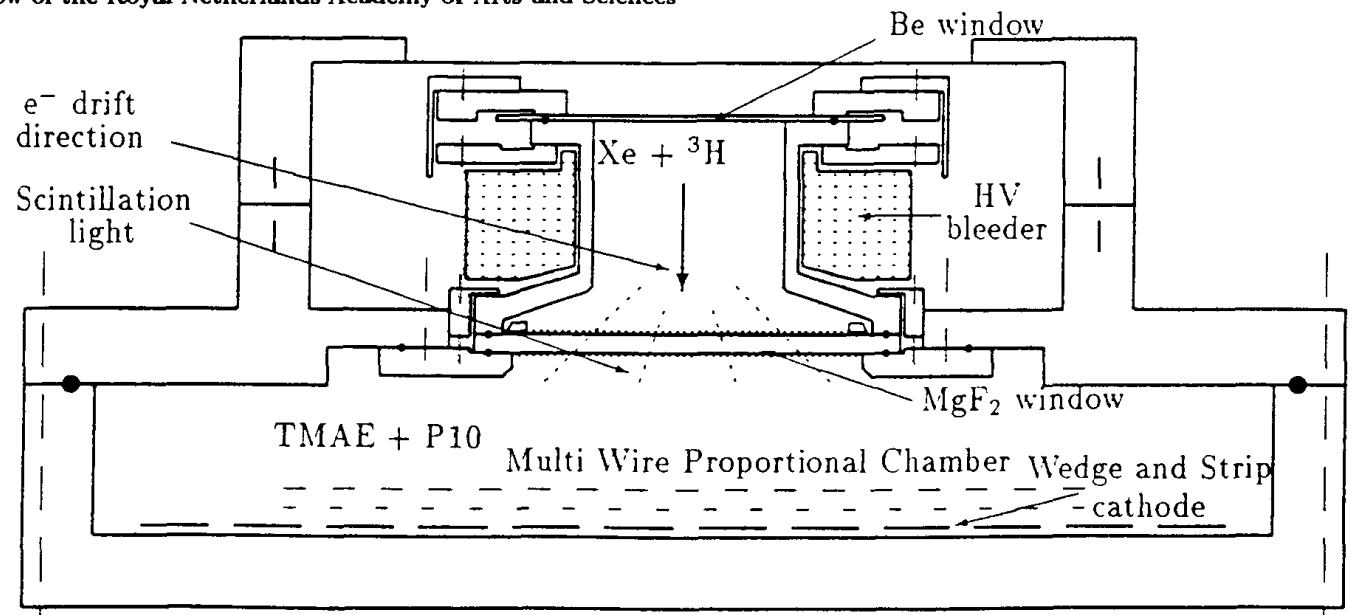

Figure 1: Schematic of the detector. 


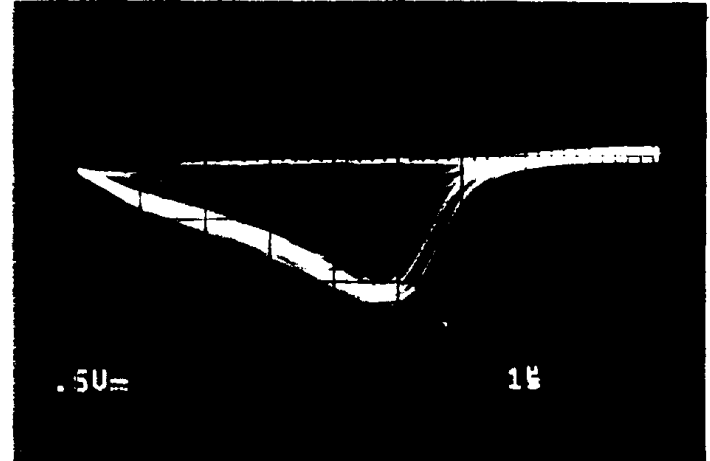

Figure 2: The output signal of the detector. The leading slope in the signal is due to the increasing solid angel as the electrons approaches the $\mathrm{MgF}_{2}$ window.

The bleeder consists of a stack of ring shaped segments made of double sided printed circuit board $(\mathrm{d}=2.4 \mathrm{~mm})$. Each segment covers one quarter of the circumference around the glass cylinder. The two copper layers of each board are interconnected by a resistor (18 $\mathrm{M} \Omega$ ) on its side. Stacking the individual pieces automatically connects the resistors from one layer to the other.

The gas scintillator body is a glass cylinder of $40 \mathrm{~mm}$ inner diameter and a length of $\mathbf{4 5} \mathrm{mm}$ with two flanges for indium seals at the top and bottom. The bottom flange has a diameter of $89 \mathrm{~mm}$ to deal with the solid angel problem [5]. At the top a $0.1 \mathrm{~mm}$ thick Be window is mounted to allow detector calibration down to about $2 \mathrm{keV}$. The Be window is kept at an HV of $-20 \mathrm{kV}$. The central part of the detector contains two grids of stainless steel (optical transparency $82 \%$ ) and a $4 \mathrm{~mm}$ thick $\mathrm{MgF}_{2}$ window. The latter has been clamped gastight between the scintillator body and a multi wire proportional chamber (MWPC) using a viton $O-$ ring and an indium seal. The grids are kept at ground potential. In the gas scintillator (operated with $\mathrm{Xe}$ ) the electrons formed by the absorption of radiation will drift towards the $\mathrm{MgF}_{2}$ window due to the applied electric field of about $5 \mathrm{kV} / \mathrm{cm}$. Each electron produces one photon $(\sim$ $165 \mathrm{~nm}$ ) per $10 \mathrm{~V}$ of traversed potential difference.A fraction of these photons is detected in the MWPC located in the lower part of the detector. The MWPC and related electronics are described in [6]. To achieve UV photon sensitivity the MWPC is flushed with TMAE and P10. In a properly operating GSPD the fraction of detected photons is bigger than one, resulting in so called light amplification. The light amplification makes the output signal less sensitive for fluctuations in the charge amplification in the MWPC. Consequently the energy resolution of the detector is essentially determined by the primary ionization process.

The MWPC is made position sensitive by means of a "wedge and strip" cathode. Position sensitivity in the drift direction is obtained by using time information included in the shape of the signal. In 1 bar of xenon the maximum track of a beta particle from tritium decay is $2 \mathrm{~mm}$, so that

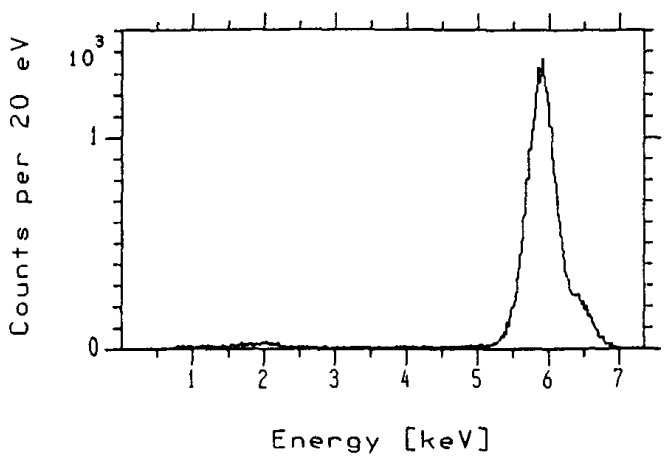

Figure 3: Spectrum of a ${ }^{55} \mathrm{Fe}$ source, illuminating the whole Be entrance window. The FWHM energy resolution is $9 \%$.

timing with a precision of about $200 \mathrm{~ns}$ will suffice in this case. In figure 2 we show the response of the detector to 6 kev $X$ rays. The absorption depth can be deduced from the different lengths of the signals. The height of the output signal is determined by the energy of the absorbed quantum. To obtain an unambiguous measure of the energy, independent of the "depth" of absorption a time window is set on the output signal corresponding to the sone between $\sim 11 \mathrm{~mm}$ and $1 \mathrm{~mm}$ from the $\mathrm{MgF}_{2}$ window. The integral light yield in this time window is the energy measure. In figure 3 the energy spectrum of ${ }^{55} \mathrm{Fe}$ is shown.

\section{The experiment}

The principle of all experiments is to search for a distortion in either a beta spectrum or a spectrum of internal bremsstrahlung in electron capture (IBEC) due to the presence of a heavy ( $17 \mathrm{keV}$ ) neutrino. The main problem in the experiments is the minuteness of the distortion [7]. In the proposed experiment, beta particles from the decay of tritium will be detected in a gas scintillation proportional detector.Xenon ( 1 bar, room temperature) is both the detection medium and the gas scintillator. The calorimetric approach is followed: xenon is mixed with a small amount of tritium.

Compared with the calorimetric semi-conductor detector experiments the GSPD experiment has the advantage that the source/detector density is approximately three orders of magnitude smaller. Clustering of tritium is not possible and solid state effects are absent.

Metal parts inside the detector are covered with a thin (300 $\mathrm{nm}$ ) gold layer to minimise sticking of tritium on the walls (which was a serious problem in a proportional counter experiment with stainless steel walls [4]). We also covered the Be window with a gold layer although $\mathrm{Be}$ is not known for tritium sticking. $\mathrm{MgF}_{2}$ is not likely to bind tritium, because it is more stable than HF. Attachment by van der Waals forces is very weak at room temperature and will 
be broken by the abound collisions of xenon atoms to the wall.

A possible wall effect from the $\mathrm{MgF}_{2}$ can be discriminated easily, since these signals will be far shorter than the required drift time for the energy measure. In addition we can eliminate the signal from decays on or near the side wall because the system is position sensitive.

\section{Spectrum distortion}

A crucial point is the linear response of the xenon system. It is known that for X-ray detection the Fano factor and the specific ionisation energy show non-linearities around the binding energies of the xenon electrons. Therefore the detector response in the region below $6 \mathrm{keV}$ has to be carefully calibrated with radioactive sources before the start of the tritium measurements. Furthermore, the response function of the detector will be measured as a function of position in order to determine $\mathrm{X}$-ray escape effects. To avoid any uncertainty with respect to the last two points the experiment will be repeated with a gas filling of argon instead of renon. Then we will have different binding energies and consequently different non-linearities and also different escape probabilities of X-rays. Once running with tritium the system must be calibrated regularly with ${ }^{55} \mathrm{Fe}$ $(6 \mathrm{keV})$ and ${ }^{109} \mathrm{Cd}(22 \mathrm{keV})$ to check the stability of the system.

Possible other sources of spectrum distortion are:

- Detected bremsstralung originated from beta particles hitting the wall (X-ray absorption)

- Bremsstralung from a beta particle which escapes from the detector (X-ray escape)

- Bremsstralung from a beta particle absorbed in the detector at a different position than the original beta decay (summation)

- Pile-up, when two separate tritium decays happen within the electron drift time in the detector.

- Characteristic wall material X-ray detection (X-ray absorption)

Most effects are not separately measurable. Simulations [7] have shown that spectrum distortion by bremsstrahlung is negligibly small compared to the $17 \mathrm{keV}$ neutrino 'finger print'. In figure 4 we show the spectrum of the distortion for one detector geometry, together with the heavy neutrino finger print for a mixing of $1 \%$.

Pile-up can be rejected by electronic signal processing. The presence of characteristic X-rays from the involved materials in the detector is made negligible in the energy region of interest by the choice of materials and by selecting a volume which is $\geq 2 \mathrm{~mm}$ off the walls.

Signals from cosmic rays can be eliminated by measuring in anti-coincidence with signals from plastic scintillators mounted above and below the GSPD or by pulse shape discrimination.

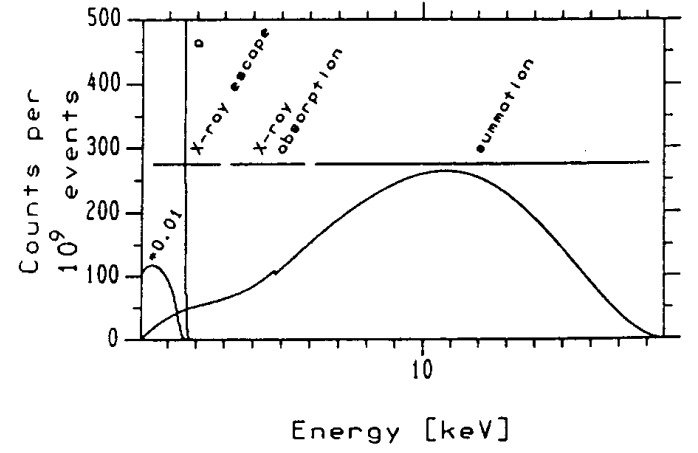

Figure 4: Simulated spectrum of the distortion for the mentioned effects and an effective volume with a diameter of $30 \mathrm{~mm}$. The dominating processes are indicated in the spectrum. The simulated contribution of the ${ }^{n} 17 \mathrm{keV}$ neutrino" is also shown (a). It is clear that the disturbing processes are negligibly small.

\section{Conclusion}

With a count rate of 1000 events per second (incl. position information), of which 300 are considered good events, it takes $\mathbf{4 0}$ days to produce the required statistics.

We have shown that the detector possess the required energy resolution and drift properties. We continue calibration and background measurements this year. Measurements with admixed tritium to the renon gas will start in 1993 and the corresponding data analysis will bring us at the end of 1994 for a final result.

\section{References}

[1] J.J. Simpson, Phys.Rev.Lett. 54 (1985) 1891-1893.

[2] B. Sadoulet, Workshop summary, Transparencies from the Workshop on the $17 \mathrm{keV}$ Neutrino Question, Berkeley, California, 18-20 December 1991.

[3] A. Ljubicic, B.A. Logan and I. Zlimen, Particle World 2 No 4 (1991) 101-107.

[4] G. Kalbfleisch and M. Bahran, preprint Univ. Oklahoma (t.b.p. in Phys. Rev. Lett.).

[5] W.J.C. Okx, C.W.E. van Eijk, R.W. Hollander and A. Zoutendijk,IEEE Transactions on Nuclear Science 34 No 6 (1987) 1818-1821.

[6] W.J.C. Okx, C.W.E. van Eijk, R. Ferreira Marques, R.W. Hollander, D. Langerveld, A. Stanovnik, C.A. Baker, J.D. Davies, J. Moir, S. Sakamoto, E.W.A. Lingeman, R.E. Welsh and R.G. Winter, IEEE Transactions on Nuclear Science 33 No 1 (1986) 391-394.

[7] W.J.C. Okx, C.W.E. van Eijk, V.R. Bom, R.W. Hollander, Nuclear Instruments and Methods in Physics Research A323(1992)466. 